\title{
A second riboswitch class for the enzyme cofactor $N A D^{+}$
}

\author{
SHANKER S.S. PANCHAPAKESAN, ${ }^{1,5}$ LUKAS COREY, $^{1,5}$ SARAH N. MALKOWSKI, ${ }^{2}$ GADARETH HIGGS, ${ }^{1}$ \\ and RONALD R. BREAKER ${ }^{1,3,4}$

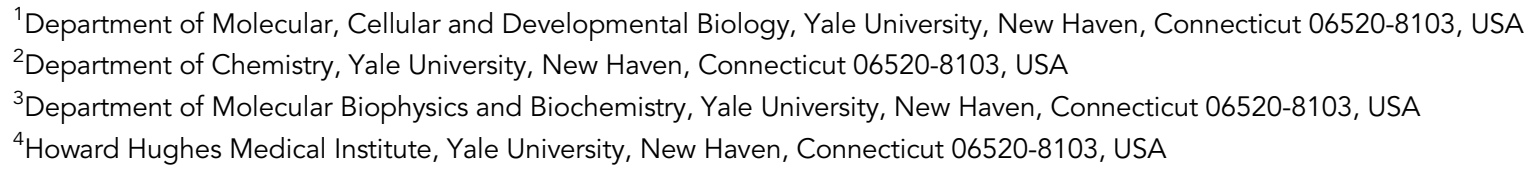

\section{ABSTRACT}

A bacterial noncoding RNA motif almost exclusively associated with pnuC genes was uncovered using comparative sequence analysis. Some PnuC proteins are known to transport nicotinamide riboside (NR), which is a component of the ubiquitous and abundant enzyme cofactor nicotinamide adenine dinucleotide (NAD ${ }^{+}$). Thus, we speculated that the newly found "pnuC motif" RNAs might function as aptamers for a novel class of NAD ${ }^{+}$-sensing riboswitches. RNA constructs that encompass the conserved nucleotides and secondary structure features that define the motif indeed selectively bind $\mathrm{NAD}^{+}$, nicotinamide mononucleotide (NMN), and NR. Mutations that disrupt strictly conserved nucleotides of the aptamer also disrupt ligand binding. These bioinformatic and biochemical findings indicate that pnuC motif RNAs are likely members of a second riboswitch class that regulates gene expression in response to NAD ${ }^{+}$binding.

Keywords: aptamer; gene control; pnuC; nicotinamide adenine dinucleotide; nicotinamide riboside transporter; noncoding RNA

\section{INTRODUCTION}

Most known riboswitch classes sense compounds that are derived from RNA monomers or their precursors (McCown et al. 2017). This finding is consistent with the hypothesis that some of these metabolite-sensing noncoding RNAs might have an ancient origin, and sense compounds that were likely relevant during the RNA World (Benner et al. 1989). For example, nucleotide-like enzyme cofactors are ligand targets for the largest collection of riboswitch aptamers, and there are at least 16 classes known to date that sense these coenzymes (McCown et al. 2017; Mirihana Arachchilage et al. 2018; Atilho et al. 2019; Chen et al. 2019; Malkowski et al. 2019).

A recent report (Malkowski et al. 2019) presented bioinformatic, genetic, and biochemical data supporting the hypothesis that nadA motif RNAs (Weinberg et al. 2017) function as riboswitches for the coenzyme $\mathrm{NAD}^{+}$. This discovery helped overcome the strange circumstance that $\mathrm{NAD}^{+}$, one of the most abundant, ubiquitous, and ancient coenzymes in biology, had no identified corresponding riboswitch classes (Breaker 2011; Malkowski et al. 2019). In contrast, nearly all other coenzymes are sensed by one

${ }^{5}$ These authors contributed equally to this work.

Corresponding author: ronald.breaker@yale.edu

Article is online at http://www.rnajournal.org/cgi/doi/10.1261/rna. 077891.120. Freely available online through the RNA Open Access option. or more distinct riboswitch classes that regulate genes related to the transport, synthesis, or use of these molecules (McCown et al. 2017).

The known $\mathrm{NAD}^{+}$-sensing riboswitches based on the nadA motif are rare (101 representatives) and limited to the phylum Acidobacteria (Malkowski et al. 2019), whereas many thousands of representatives are known for some riboswitch classes that sense other coenzymes (McCown et al. 2017). The relative scarcity of riboswitches based on the nadA motif could be explained by two possibilities. First, modern cells might have little need to directly monitor $\mathrm{NAD}^{+}$concentrations because they use other mechanisms to adjust metabolic pathways in response to changing concentrations of this enzyme cofactor. However, this seems highly unlikely due to the great importance of $\mathrm{NAD}^{+}$in many metabolic pathways spanning biosynthesis, degradation, and energy management processes (Clarke and Dafforn 1998). Second, it is possible that the task of sensing $\mathrm{NAD}^{+}$is distributed over multiple different systems, including diverse protein factors, and possibly also by additional $\mathrm{NAD}^{+}$riboswitch classes that remain to be discovered (Malkowski et al. 2019).

Given the importance of NAD ${ }^{+}$and related compounds, and given the dearth of known riboswitches for this

(C) 2021 Panchapakesan et al. This article, published in RNA, is available under a Creative Commons License (Attribution 4.0 International), as described at http://creativecommons.org/licenses/by/4.0/. 
coenzyme, we were particularly intrigued by the discovery of a structured RNA domain that we named the "pnuC motif" (KI Brewer, EB Greenlee, G Higgs, et al., in prep.). This name was chosen because representatives commonly reside in the $5^{\prime}$ untranslated regions of open reading frames annotated as pnuC. These genes code for membrane proteins that presumably transport the nucleoside derivative nicotinamide riboside (NR) (Zhu et al. 1989; Kemmer et al. 2001; Sauer et al. 2004). Thus, the pnuC motif consensus might reflect the essential features of an aptamer domain for a novel riboswitch class that senses $\mathrm{NAD}^{+}$or a related compound.

In this report, we present bioinformatic and biochemical data indicating that pnuC motif RNAs function as riboswitches selective for $\mathrm{NAD}^{+}$and its NR-containing fragments. RNA constructs carrying the consensus pnuC motif undergo substantial folding changes that are induced only by their cognate ligands. Biochemical analysis of analogs indicates that the aptamer selectively recognizes functional groups on the nicotinamide-derived nucleobase. This latter finding demonstrates that natural RNAs directly recognize the redox-active moiety of this coenzyme and its immediate precursors. These findings add to the number and functional diversity of bacterial RNAs that monitor the levels of the ubiquitous coenzyme NAD ${ }^{+}$.

\section{RESULTS AND DISCUSSION}

\section{A riboswitch candidate called the pnuC motif is associated with nicotinamide riboside transport}

A novel riboswitch candidate called the pnuC motif (Fig. 1A) was discovered (KI Brewer, EB Greenlee, G Higgs, et al., in prep.) by using a comparative sequence analysis approach that comprehensively reveals novel structured RNA motifs within bacterial genomes of interest. Specifically, the motif was uncovered by analyzing the sequences of noncoding regions of a given genome that are both unusually long and biased in favor of $G$ and C nucleotides (Meyer et al. 2009; Stav et al. 2019). RNAs such as riboswitches rarely overlap protein coding regions, and usually use plentiful G and $C$ nucleotides to form their aptamer structures even when present in bacterial species that have strongly AT-rich genomes (Klein et al. 2002; Schattner 2002; Meyer et al. 2009;
Stav et al. 2019). These long, GC-rich genomic regions are examined for evidence of sequence and structural similarity to the noncoding regions of all bacterial genomes. Initially, we identified approximately 130 representatives of a conserved region commonly located upstream of a gene called pnuC, exclusively from species in the bacterial genus Streptococcus. A second gene frequently annotated as "hypothetical" is also frequently associated with pnuC motif representatives. The function of the protein expressed by this gene is unclear, and its presence usually coincides with a transposase gene, which further obscures its biological relevance to the RNA motif. Therefore our list of ligand candidates was inspired only by the predicted function of PnuC proteins as discussed below.

The consensus model for the pnuC motif (Fig. 1A) was derived by examining 43 unique-sequence representatives spanning only the most prominently conserved region (Supplemental Fig. S1). Given the small number of highly similar examples, most nucleotide positions appear to be highly conserved. Although we predict that three base-paired substructures are formed, called P1, $\mathrm{P} 1 \mathrm{a}$, and a possible pseudoknot (pk), only stem P1a is
A pnuC RNA Motif

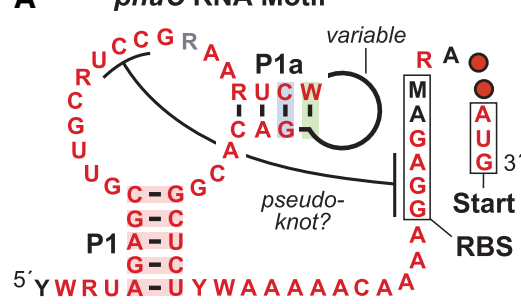

Nucleotide identity

N $97 \% \quad$ N $75 \%$

N $90 \%$

Nucleotide present

. $97 \%$ O $75 \%$

$90 \%$ O $50 \%$

C 0.330

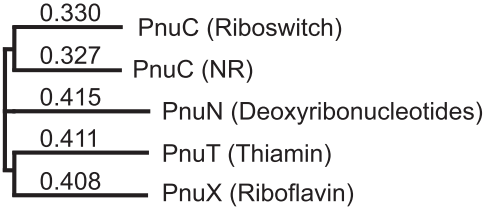

$R=A / G \quad Y=C / U$

$\mathrm{W}=\mathrm{A}$ or $\mathrm{U} \mathrm{M}=\mathrm{A}$ or $\mathrm{C}$

covariation

compatible mutations

no mutations

eotides)
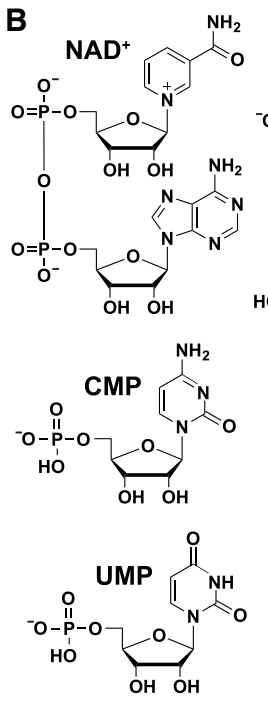

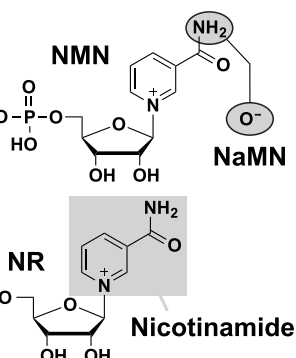

$\mathrm{OH}$

$\boldsymbol{B}=$ ADP-ribosyl

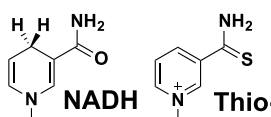

R

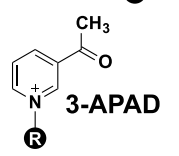

FIGURE 1. The pnuC motif and candidate riboswitch ligands. (A) Consensus sequence and secondary structure model for pnuC motif RNAs. The consensus was generated based on the comparison of 43 representatives with distinct sequences spanning the putative aptamer region (Supplemental Fig. S1). The boxed nucleotides near the $3^{\prime}$ end identify nucleotides serving as the ribosome binding site (RBS) and start codon. (B) Chemical structures of NAD ${ }^{+}$ and various natural or synthetic analogs. 3-APAD is 3-acetylpyridine adenine dinucleotide. (C) Comparison of the amino acid sequences of five proteins by Clustal Omega: PnuC associated with a pnuC motif RNA from Streptococcus sp. HMSC070B10, a PnuC from Haemophilus influenzae proven to transport NR (Herbert et al. 2003), a PnuT thiamin transporter (Jaehme et al. 2018), a PnuX riboflavin transporter from Corynebacterium glutamicum (Vogl et al. 2007), and a PnuN protein from Lactobacillus acidophilus predicted to transport a deoxynucleoside (Rodionov and Gelfand 2005). Depicted is an unrooted phylogenetic tree indicating that the riboswitch-associated PnuC protein is most homologous to a PnuC protein known to transport NR. The numbers are unitless representations of genetic distance (lengths of horizontal lines), and indicate the number of substitutions as a proportion of the alignment length. 
supported by evidence of nucleotide covariation consistent with base-pairing. This stem also appears to carry a variable loop region whose sequence is not important for the function of the RNA. Although the proposed P1 and pseudoknot substructures lack evidence of covariation, these structures are consistent with structural probing data as described in detail in a later section.

PnuC proteins encoded by pnuC genes associated with the newly discovered RNA motif are annotated as transporters for NR (e.g., see Zhu et al. 1989; Kemmer et al. 2001; Sauer et al. 2004). NR is a component of the ubiquitous enzyme cofactor nicotinamide adenine dinucleotide (NAD ${ }^{+}$) (Fig. 1B). Also, the possible pk interaction involves nucleotides predicted to serve as the ribosome binding site (RBS) for the adjacent coding region. This suggests that pnuC motif RNAs are likely to be genetic "OFF" riboswitches. Therefore, we speculated that the pnuC motif might represent the conserved aptamer domain of a novel riboswitch class that senses and responds to a molecule in the NAD ${ }^{+}$biosynthetic pathway. However, various homologs of PnuC proteins have been proven to transport other metabolites such as thiamin (PnuT) (Jaehme et al. 2018) and riboflavin (PnuX) (Vogl et al. 2007), or have been proposed to transport deoxyribonucleosides (PnuN) (Rodionov and Gelfand 2005). The existence of these other proteins left open the possibility that the pnuC genes associated with this novel RNA motif were misannotated.

To assess this possibility, we conducted an analysis of the amino acid sequences of representative transporter proteins in this collection. Multiple sequence analysis using Clustal Omega (Sievers et al. 2011) revealed that the amino acid sequence of a randomly chosen PnuC protein associated with the newly found RNA motif (from Streptococcus sp. HMSC070B10) was most similar to a protein proven to selectively transport NR (Vogl et al. 2007) compared to the other transporter types (Fig. 1C). Similar results were observed by implementing an analysis using protein BLAST (Supplemental Fig. S2).

Indeed, some of the more distantly related PnuT and PnuX proteins are the products of genes occasionally found associated with riboswitches for TPP and FMN, respectively (data not shown). Thus, the specificities of the PnuC-like transporters can be predicted by the riboswitch class regulating their respective genes, and therefore it is logical that genes coding for NR transporters would be associated with a riboswitch class for $\mathrm{NAD}^{+}$. Therefore, we focused our biochemical analyses on assessing the ligand binding function of $\mathrm{NAD}^{+}$and its derivatives with constructs carrying the pnuC motif consensus.

\section{RNA constructs carrying a pnuC motif bind NAD ${ }^{+}$ and related compounds}

Initial RNA constructs used to assess ligand binding function were prepared from a representative pnuC motif present in Streptococcus parasanguinis. RNA constructs called 85 pnuC or 65 pnuC (Fig. 2A) carrying either 85 or $65 \mathrm{nt}$ of the natural genomic sequence, respectively, were synthesized by in vitro transcription. These were $5^{\prime 32} \mathrm{P}$-labeled, and subsequently subjected to in-line probing analysis (Soukup and Breaker 1999; Regulski and Breaker 2008) to monitor changes in RNA structure brought about by ligand binding. The lengths of these constructs were chosen to evaluate the importance of nucleotides located immediately upstream of the proposed P1 stem, which was of interest due to the possible presence of conserved nucleotides.

In-line probing reactions conducted with 85 pnuC RNA revealed that $\mathrm{NAD}^{+}$and $\mathrm{NMN}$ both induce substantial changes in the pattern of spontaneous RNA strand scission (Fig. 2B), which is indicative of structural reorganization brought about by ligand binding. Furthermore, no modulation is observed when the enzyme cofactors thiamin pyrophosphate (TPP) or flavin mononucleotide (FMN) are introduced (Supplemental Fig. S3). These results are consistent with the hypothesis that PnuC proteins associated with pnuC motif representatives are most likely NR transporters and that gene expression should be regulated by a compound indicative of the status of cellular NAD ${ }^{+}$ concentration.

The structural changes induced by $\mathrm{NAD}^{+}$and NMN only begin with nucleotides forming the junction between P1 and $\mathrm{P} 1 \mathrm{a}$ of the 85 pnuC construct (Fig. 2A), suggesting that the first $\sim 30 \mathrm{nt}$ of the 85 pnuC RNA construct are not important for ligand binding. Similarly, in-line probing reactions conducted with the 65 pnuC RNA also exhibit robust changes to the banding pattern with $\mathrm{NAD}^{+}, \mathrm{NMN}$, and NR, but not with nicotinic acid (NA) (Supplemental Fig. S4). Both constructs exhibit a pattern of bands from in-line probing reactions that are consistent with the predicted base-pairing interactions, including the pk substructure. These results suggest that the aptamer domain is confined to the most highly conserved part of the RNA. Both the nicotinamide and ribose moieties appear to carry the most important contacts for recognition by the aptamer. Moreover, the structure formed in the presence of $\mathrm{NAD}^{+}$is consistent with a riboswitch mechanism wherein the ribosome binding site (RBS) is sequestered to suppress gene expression when ligand is bound (Fig. 1A).

We subjected the 65 pnuC RNA construct to in-line probing analysis under various concentrations of $\mathrm{NAD}^{+}$ to establish the apparent dissociation $\left(K_{D}\right)$ value for the RNA-cofactor interaction (Fig. 2C). The fraction of RNA bound to ligand was estimated by quantifying the band intensities at several key sites. By plotting fraction bound versus the logarithm of ligand concentration, a curve that is typical of a one-to-one binding interaction resulted, with a $K_{\mathrm{D}}$ value of approximately $100 \mu \mathrm{M}$ (Fig. 2D). Similar results were observed for the 85 pnuC construct 


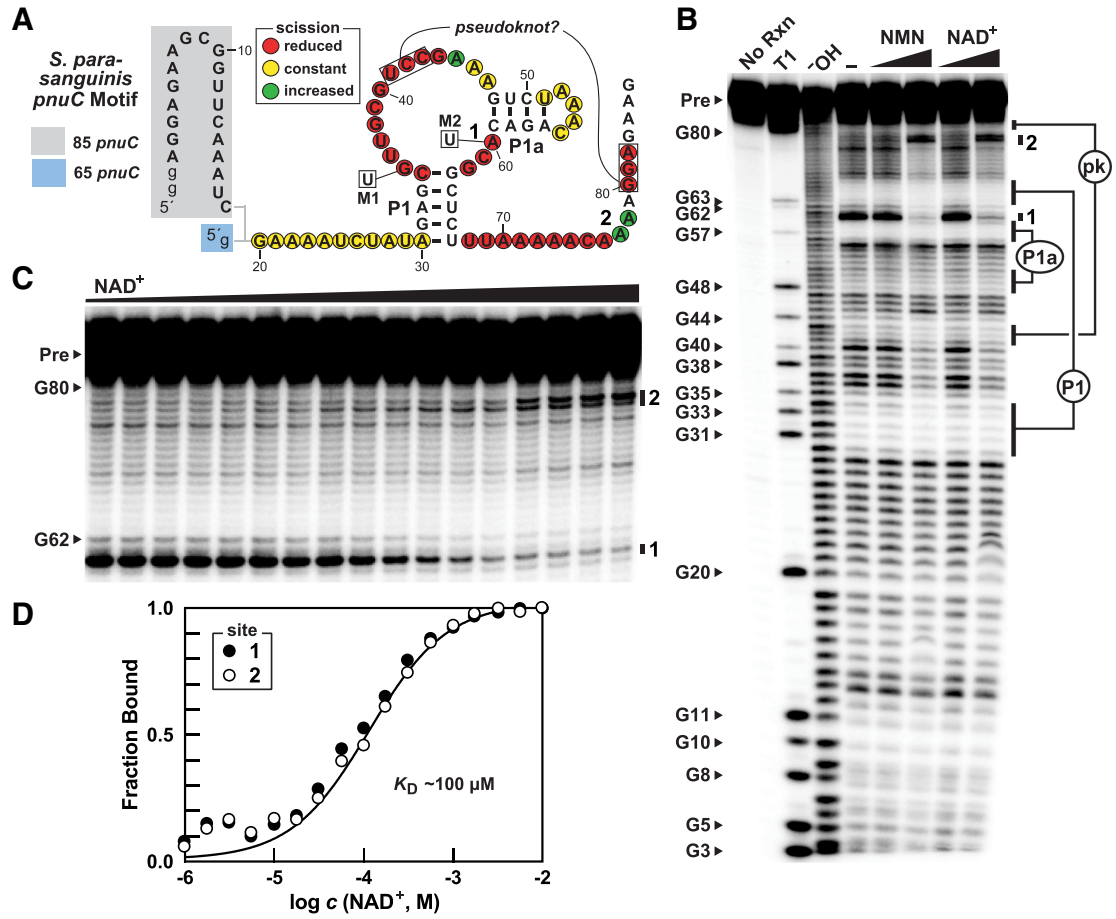

FIGURE 2. Aptamer function by pnuC RNA constructs. (A) Sequence and proposed secondary structure of RNA constructs based on the pnuC motif from $S$. parasanguinis. The distinct $5^{\prime}$ ends of the 85 pnuC and 65 pnuC constructs are highlighted, wherein the lowercase " $g$ " letters denote guanosine nucleotides added to facilitate preparation by in vitro transcription. Colored circles identify the patterns of spontaneous RNA strand scission for nucleotides 20 through 80 resulting from in-line probing assays with the 85 pnuC construct as depicted in $B$. Nucleotide changes made to create mutant constructs $M 1$ and $M 2$ are depicted in boxes. (B) PAGE analysis of in-line probing reactions conducted with $5^{\prime 2} \mathrm{P}$-labeled 85 pnuC construct RNAs. No Rxn, $\mathrm{T} 1$ and ${ }^{-} \mathrm{OH}$ identify RNAs subjected to no reaction, partial digestion with RNase T1 (cleaves after G nucleotides), or to alkali conditions (cleaves after each nucleotide), respectively. The full-length precursor RNA (Pre) and various RNase T1 cleavage products are annotated. Nucleotide regions involved in predicted base-paired structures are annotated P1, P1a, and pk (pseudoknot). Ligands were added to a final concentration of 0.1 or $1 \mathrm{mM}$. (C) PAGE analysis of in-line probing reactions conducted with $5^{\prime 32} \mathrm{P}$-labeled 65 pnuC construct RNAs with concentrations of $\mathrm{NAD}^{+}$ranging from $1 \mu \mathrm{M}$ to $10 \mathrm{mM}$ to represent each $0.25 \log \mathrm{M}$ concentration units. (D) Plot of the fraction of RNA bound to ligand versus the logarithm of the ligand concentration (c). Values for fraction bound were estimated from the data in $C$ based on the ligand intensities at the sites indicated. Other annotations are as described in $B$. domains for a novel $\mathrm{NAD}^{+}$riboswitch class that we are naming $\mathrm{NAD}^{+}-\mathrm{Il}$, given that another class (hereafter called $\mathrm{NAD}^{+}$-I) for this coenzyme also has been published recently (Malkowski et al. 2019).

\section{$\mathrm{A} \mathrm{NAD}^{+}$-II riboswitch aptamer requires conserved nucleotides to selectively recognize the nicotinamide riboside domain of $\mathrm{NAD}^{+}$}

To further explore the importance of conserved nucleotides and the molecular determinants for ligand binding, we examined a series of additional RNA constructs and compounds for evidence of binding. We first determined whether single-nucleotide mutations (Fig. 2A), arbitrarily chosen from the strictly conserved positions observed (Fig. 1A), would disrupt ligand binding by the 65 pnuC RNA. Indeed, mutant RNA constructs M1 (G35U) and M2 (A60U) fail to respond to $1 \mathrm{mM} \mathrm{NMN} \mathrm{(Fig.} \mathrm{3A).}$ In contrast, the wild-type WT 65 pnuC RNA construct exhibits the expected changes in banding pattern generated by in-line probing. Given the rarity of this riboswitch class, however, we cannot be certain at this time that all nucleotide positions with conserved sequence identity are essential for ligand binding function.

Next, we screened additional $\mathrm{NAD}^{+}$analogs for ligand binding function. Of particular interest were those analogs that carry chemical (Supplemental Fig. S5), suggesting again that the shorter construct carries the complete aptamer domain. There is no indication from this data that the riboswitch aptamer senses the adenosyl moiety of $\mathrm{NAD}^{+}$.

The concentration of $\mathrm{NAD}^{+}$, which is the predominant biochemical form of the nicotinamide moiety, has been measured at $\sim 2.6 \mathrm{mM}$ in Escherichia coli cells (Bennett et al. 2009). Therefore, the aptamer has an affinity for $\mathrm{NAD}^{+}$that is more than two orders of magnitude better than that needed if it were to function as a genetic switch at thermodynamic equilibrium. This suggests that pnuC motif RNAs might function as kinetically driven riboswitches (Wickiser et al. 2005a,b; Gilbert et al. 2006; Haller et al. 2011; Lemay et al. 2011; Frieda and Block 2012). Thus, pnuC motif RNAs appear to serve as aptamer changes to the nicotinamide moiety, which appears to be necessary for ligand binding (Supplemental Figs. S3, S4). The 65 pnuC RNA construct strongly discriminates against all compounds tested that carry a modification to the nicotinamide ring (Fig. 3B), including the reduced form of the natural coenzyme (NADH), or a change to the amide moiety of the pyridine ring of nicotinamide (Thio-NAD ${ }^{+}$, 3-APAD, and NaMN) (Fig. 1B). The RNA also rejects the pyrimidine nucleotides $5^{\prime}-\mathrm{CMP}$ and $5^{\prime}$ UMP, which is important to prevent erroneous gene regulation given that these molecules and their various phosphorylated derivatives are abundant in cells (Bennett et al. 2009). As expected, inversion of the anomeric center formed between the nicotinic acid and ribose moieties, as present in $\alpha-\mathrm{NAD}^{+}$, also causes a loss of ligand binding. 
A
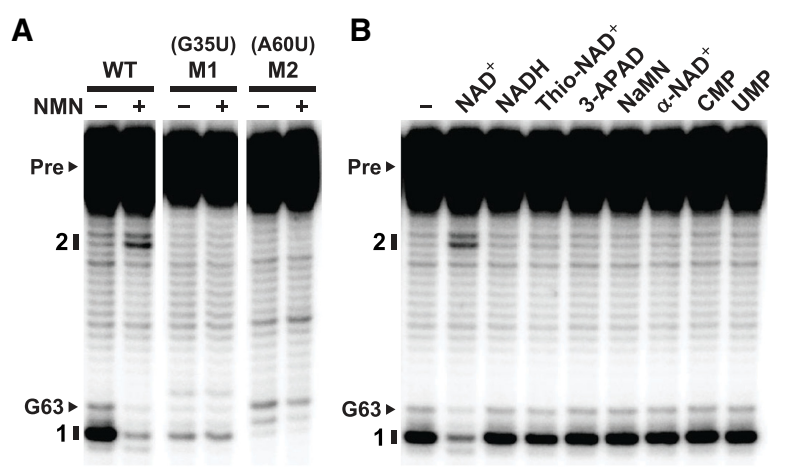

C

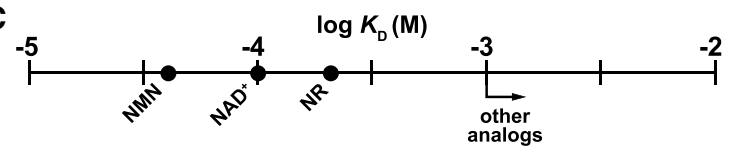

D

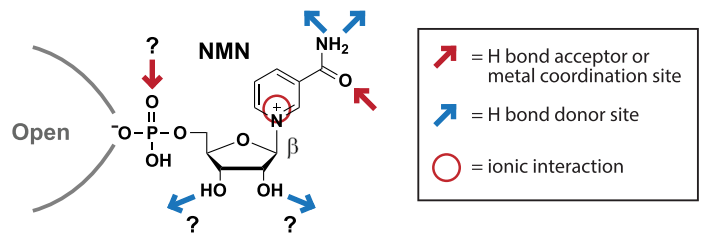

FIGURE 3. Components of the RNA and ligand essential for molecular recognition. (A) In-line probing assays with WT pnuC 65 RNA, and with mutants M1 (G35U) and M2 (A60U) in the absence (-) or presence $(+)$ of $1 \mathrm{mM}$ NMN. Additional annotations are as described for Figure 2B. (B) In-line probing assays with WT pnuC 65 RNA in the absence (-) of ligand, or in the presence of $1 \mathrm{mM}$ of the compounds noted. (C) Plot of the $K_{\mathrm{D}}$ values determined for $\mathrm{NAD}^{+}$and various analogs. Note that all compounds tested, except for $\mathrm{NAD}^{+}, \mathrm{NMN}$, and NR, have $K_{D}$ values poorer than $1 \mathrm{mM}$. (D) Model depicting possible ligand-binding interactions form $\mathrm{NAD}^{+}$-II riboswitch aptamers based on the existing data.

Given the apparent lack of contributions to binding affinity by the adenosyl moiety of $\mathrm{NAD}^{+}$, and given the disruptive effects of even modest changes to the nicotinamide ring of the ligand, we did not test AMP, ADP, or ATP for binding activity

These findings (Fig. 3B), along with those discussed earlier (Supplemental Figs. S3. S4) indicate that the aptamer of $\mathrm{NAD}^{+}$-II riboswitches selectively recognizes the nicotinamide moiety joined to a ribose via a $\beta$-N-glycosidic linkage. In-line probing assays conducted at various concentrations of ligand were used to establish $K_{D}$ values for the three compounds that exhibit binding activity (e.g. see Fig. 2C). These values (Fig. $3 \mathrm{C}$ ) indicate that the $5^{\prime}$ AMP moiety of $\mathrm{NAD}^{+}$likely does not contribute to the molecular recognition process of $\mathrm{NAD}^{+}-\mathrm{Il}$ riboswitch aptamers. Thus, we speculate that the RNA forms a precise binding pocket for NR recognition, and that the $5^{\prime}$-AMP portion of $\mathrm{NAD}^{+}$will protrude outward from the aptamer core and be exposed to solvent (Fig. 3D). This is opposite of the configuration observed for the first aptamer domain of $\mathrm{NAD}^{+}$-I riboswitches (Malkowski et al. 2019), which selectively binds the $5^{\prime}$-ADP moiety and leaves the nicotinamide moiety exposed to solvent (Huang et al. 2020).

\section{Concluding remarks}

Our findings indicate that pnuC motif RNAs are selective sensors of NR or its $5^{\prime}$-modified derivatives such as NMN and $\mathrm{NAD}^{+}$. Given the fact that the $\mathrm{NAD}^{+}$concentration measured from $E$. coli cells is vastly greater than other natural metabolites carrying a nicotinamide mononucleotide moiety (Bennett et al. 2009), the biologically relevant ligand for regulating gene expression appears to be $\mathrm{NAD}^{+}$. However, because other nicotinamide-containing compounds are also recognized, cells likely use $\mathrm{NAD}^{+}-$II riboswitches to measure the pool of natural NR derivatives and activate expression of the pnuC gene if these levels become too low.

The surprising exception to this ligand collection is the reduced form of the coenzyme, NADH. Perhaps the RNA aptamer exploits the positive charge in the oxidized nicotinamide ring as a molecular recognition contact (Fig. 3D). This could also explain the requirement for the ribose moiety, whose absence would eliminate the positive charge in the pyridine ring. Given this possibility, we cannot be certain that the hydroxyl groups of the ribose moiety are exploited by the aptamer as hydrogen bond donor contacts. Identifying the precise molecular recognition with greater certainty is likely to result from biophysical analyses that establish the atomic-resolution structure of the aptamer-ligand complex, as has been achieved for an $\mathrm{NAD}^{+}$-I riboswitch aptamer (Huang et al. 2020).

$\mathrm{NAD}^{+}$-II riboswitches constitute the second class that responds to this coenzyme. The first class to be reported, based on the nadA motif (Weinberg et al. 2017) and now called $\mathrm{NAD}^{+}$-I riboswitches, uses a very unusual architecture where the adenosine monophosphate (AMP) and NMN moieties appear to be bound by separate but similar aptamer domains arranged in tandem (Malkowski et al. 2019; Huang et al. 2020). The AMP moiety is selectively recognized by the first aptamer domain, whereas the NMN moiety is exposed on the surface of the aptamer. Although it has yet to be demonstrated that the nicotinamide moiety of $\mathrm{NAD}^{+}$is selectively recognized by the second domain of $\mathrm{NAD}^{+}-1$ riboswitches, this outcome seems likely due to the similarity between the tandem aptamer domains, the similarity in the chemical structures of AMP and NMN, and the fact that the NMN moiety is exposed to solvent when the AMP moiety is docked to the first aptamer.

However, there is no notable similarity between the second aptamer domain of $\mathrm{NAD}^{+}-1$ riboswitches and the aptamer domain of $\mathrm{NAD}^{+}-\|$riboswitches, suggesting that these RNAs will recognize the nicotinamide moiety using distinct binding pockets. In the current report, we establish that the aptamers of $\mathrm{NAD}^{+}$-II riboswitches indeed make direct contacts with the NR portion of the enzyme cofactor, which is required for the RNAs to distinguish $\mathrm{NAD}^{+}$from all other compounds in the cell that carry an 
adenosyl moiety. Indeed, it might be advantageous for $\mathrm{NAD}^{+}$-II riboswitch aptamers to ignore the adenosyl moiety, which would allow the RNA to avoid being triggered by the many high-concentration compounds in cells that carry this same moiety.

The discovery and validation of $\mathrm{NAD}^{+}-\mathrm{II}_{\text {riboswitches }}$ aids efforts to determine the function of proteins whose expression is regulated by these riboswitches. Given that PnuC-like proteins have diversified to transport various metabolites (Jaehme and Slotboom 2015), riboswitch associations can help in the process of defining the ligands for these proteins. Our findings also support the hypothesis that modern cells might use numerous different RNA architectures to sense the enzyme cofactor $\mathrm{NAD}^{+}$. This would help explain why a more abundantly represented riboswitch class has not been discovered for $\mathrm{NAD}^{+}$, but have for some other types of enzyme cofactors such as coenzyme $B_{12}$, TPP, SAM, FMN, and others (McCown et al. 2017). If this is true, then additional rare riboswitch classes with distinct $\mathrm{NAD}^{+}$-binding aptamers are likely to be discovered in the future.

\section{MATERIALS AND METHODS}

\section{Chemicals}

All chemicals were purchased from Sigma-Aldrich except for $\left[\gamma-{ }^{32} \mathrm{P}\right]-A T P$, which was purchased from PerkinElmer. Enzymes and oligonucleotide sources are described elsewhere.

\section{Bioinformatic analysis of pnuC motif RNAs}

The collection of 130 pnuC motif representatives reported elsewhere (KI Brewer, EB Greenlee, G Higgs, et al., in prep.) were reduced to 43 unique representatives that were bounded by the nucleotides beginning near the left shoulder of P1 and the start codon. Alignments were manually prepared and analyzed to establish the consensus sequence and structural model. Given the small number of unique representatives, base-pair annotations were made if any evidence of covariation was observed, without statistical analysis.

\section{Bioinformatic analysis of PnuC-like proteins}

Clustal Omega was used to conduct comparative sequence analyses of a PnuC protein associated with the pnuC riboswitch candidate (Streptococcus sp. HMSC070B10) in comparison to transporters for NR (PnuC (Nicotinamide Riboside); Haemophilus influenzae), deoxynucleosides (PnuN; Lactobacillus acidophilus), thiamin (PnuT; Shewanella woodyi), and riboflavin (PnuX; Corynebacterium glutamicum). An unrooted phylogenetic tree was established based on the resulting sequence alignments.

Pairwise analyses of the sequences of the same proteins were also conducted by using BLASTP (McGinnis and Madden 2004). A FASTA file of the PnuC protein sequence associated with a
pnuC motif RNA served as the query, which was compared pairwise with all other protein sequences (Supplemental Fig. S2).

\section{RNA oligonucleotide preparation}

RNA oligonucleotides were prepared as previously described (Malkowski et al. 2019) using the appropriate synthetic DNA (Integrated DNA Technologies) templates for in vitro transcription (Supplemental Fig. S6). Template DNAs were hybridized to single-stranded T7 RNA polymerase promoter strands in transcription reactions, which were incubated at $37^{\circ} \mathrm{C}$ for $2-3 \mathrm{~h}$. The resulting RNA transcripts were separated by using denaturing (8 M urea) 10\% polyacrylamide gel electrophoresis (PAGE). RNAs were recovered from the gel, dephosphorylated using rAPid alkaline phosphatase (Roche Applied Science), and subsequently $5^{\prime 32} \mathrm{P}$-labeled using $\left[\gamma_{-}{ }^{32} \mathrm{P}\right]-\mathrm{ATP}$ and T4 polynucleotide kinase (New England Biolabs) according to the manufacturer's protocols.

\section{In-line probing analyses}

In-line probing reactions were performed as previously described (Soukup and Breaker 1999; Regulski and Breaker 2008). ${ }^{32} \mathrm{P}$-labeled RNAs (trace) were incubated in the absence or presence of ligand candidates as indicated in the presence of $20 \mathrm{mM}$ $\mathrm{MgCl}_{2}, 100 \mathrm{mM} \mathrm{KCl}$, and $50 \mathrm{mM}$ Tris- $\mathrm{HCl}\left(\mathrm{pH} 8.3\right.$ at $\left.\sim 23^{\circ} \mathrm{C}\right)$. The reaction products were separated by denaturing ( $8 \mathrm{M}$ urea) $10 \%$ PAGE and were visualized by using a Typhoon phosphorimager (GE Healthcare). As described previously (Malkowski et al. 2019), band intensities were determined and used to estimate the fraction of RNAs bound to ligand. Values were plotted relative to the logarithm of the molar concentration of ligand, wherein half-maximal binding represents the $K_{\mathrm{D}}$.

\section{SUPPLEMENTAL MATERIAL}

Supplemental material is available for this article.

\section{ACKNOWLEDGMENTS}

We thank Dr. Kimberly Harris for advice regarding protein comparisons and Drs. Harris, Adam Roth, and Hubert Salvail for comments on the manuscript. S.N.M. was supported by the National Science Foundation Graduate Research Fellowship Program (DGE1122492). This work was also supported by National Institutes of Health grants (GM022778 and Al136794) to R.R.B. Research in the Breaker laboratory is also supported by the Howard Hughes Medical Institute.

Received September 23, 2020; accepted October 19, 2020.

\section{REFERENCES}

Atilho RM, Mirihana Arachicilage G, Greenlee EB, Knecht KM, Breaker RR. 2019. A bacterial riboswitch class for the thiamin precursor HMP-PP employs a terminator-embedded aptamer. Elife 8: e45210. doi:10.7554/eLife.45210 
Benner SA, Ellington AD, Tauer A. 1989. Modern metabolism as a palimpsest of the RNA world. Proc Natl Acad Sci 86: 7054-7058. doi:10.1073/pnas.86.18.7054

Bennett BD, Kimball EH, Gao M, Osterhout R, Van Dien SJ, Rabinowitz JD. 2009. Absolute metabolite concentrations and implied enzyme active site occupancy in Escherichia coli. Nat Chem Biol 5: 593-599. doi:10.1038/nchembio.186

Breaker RR. 2011. Prospects for riboswitch discovery and analysis. Mol Cell 43: 867-879. doi:10.1016/j.molcel.2011.08.024

Chen X, Mirihana Arachchilage G, Breaker RR. 2019. Biochemical validation of a second class of tetrahydrofolate riboswitches in bacteria. RNA 25: 1091-1097. doi:10.1261/rna.071829.119

Clarke AR, Dafforn TR. 1998. NAD ${ }^{+}$and NADP-linked reactions. In Comprehensive biological catalysis (ed. Sinnott M), Vol. III, pp. 1-76. Academic Press, New York.

Frieda KL, Block SM. 2012. Direct observation of cotranscriptional folding in an adenine riboswitch. Science 338: 397-400. doi:10 $.1126 /$ science. 1225722

Gilbert SD, Stoddard CD, Wise SJ, Batey RT. 2006. Thermodynamic and kinetic characterization of ligand binding to the purine riboswitch aptamer domain. J Mol Biol 359: 754-768. doi:10.1016/j .jmb.2006.04.003

Haller A, Souliére MF, Micura R. 2011. The dynamic nature of RNA as a key to understanding riboswitch mechanisms. Acc Chem Res 44: 1339-1348. doi:10.1021/ar200035g

Herbert M, Sauer E, Smethurst G, Kraiß A, Hilpert A, Reidl J. 2003. Nicotinamide ribosyl uptake mutants in Haemophilus influenzae. Infect Immun 71: 5398-5401. doi:10.1128/IAI.71.9.5398-5401 .2003

Huang L, Wang J, Lilley D. 2020. Structure and ligand binding of the ADP-binding domain of the NAD ${ }^{+}$riboswitch. RNA 26: 878-887. doi:10.1261/rna.074898.120

Jaehme M, Slotboom DJ. 2015. Structure, function, evolution, and application of bacterial Pnu-type vitamin transporters. Biol Chem 396: 955-966. doi:10.1515/hsz-2015-0113

Jaehme M, Singh R, Garaeva AA, Duurkens RH, Slotboom DJ. 2018. PnuT uses a facilitated diffusion mechanism for thiamine uptake. J Gen Physiol 150: 41-50. doi:10.1085/jgp.201711850

Kemmer G, Reilly TJ, Schmidt-Brauns J, Zlotnik GW, Green BA, Fiske MJ, Herbert M, Kraiss A, Schlör S, Smit A, et al. 2001. NadN and e (P4) are essential for utilization of NAD and nicotinamide mononucleotide but not nicotinamide riboside in Haemophilus influenzae. J Bacteriol 183: 3974-3981. doi:10.1128/JB.183.13.3974-3981 .2001

Klein RJ, Misulovin Z, Eddy SR. 2002. Noncoding RNA genes identified in AT-rich hyperthermophiles. Proc Natl Acad Sci 99: 75427547. doi:10.1073/pnas.112063799

Lemay J-F, Desnoyers G, Blouin S, Heppell B, Bastet L, St-Pierre P, Massé $E$, Lafontaine DA. 2011. Comparative study between transcriptionally- and translationally-acting adenine riboswitches reveals key differences in riboswitch regulatory mechanisms. PLoS Genet 7: e1001278. doi:10.1371/journal.pgen.1001278

Malkowski SN, Spencer TCJ, Breaker RR. 2019. Evidence that the nadA motif is a bacterial riboswitch for the ubiquitous enzyme cofactor NAD ${ }^{+}$. RNA 25: 1616-1627. doi:10.1261/rna.072538.119

McCown PJ, Corbino KA, Stav S, Sherlock ME, Breaker RR. 2017. Riboswitch diversity and distribution. RNA 23: 995-1011. doi:10 $.1261 /$ rna.061234.117
McGinnis S, Madden TL. 2004. BLAST: at the core of a powerful and diverse set of sequence analysis tools. Nucleic Acids Res 32: W20W25. doi:10.1093/nar/gkh435

Meyer MM, Ames TD, Smith DP, Weinberg Z, Schwalbach MS, Giovannoni SJ, Breaker RR. 2009. Identification of candidate structured RNAs in the marine organism 'Candidatus Pelagibacter ubique'. BMC Genomics 10: 268. doi:10.1186/1471-2164-10-268

Mirihana Arachchilage G, Sherlock ME, Weinberg Z, Breaker RR. 2018. SAM-VI RNAs selectively bind S-adenosylmethionine and exhibit similarities to SAM-III riboswitches. RNA Biol 15: 371378. doi:10.1080/15476286.2017.1399232

Regulski EE, Breaker RR. 2008. In-line probing analysis of riboswitches. Methods Mol Biol 419: 53-67. doi:10.1007/978-159745-033-1_4

Rodionov DA, Gelfand MS. 2005. Identification of a bacterial regulatory system for ribonucleotide reductases by phylogenetic profiling. Trends Genet 21: 385-389. doi:10.1016/j.tig.2005.05.011

Sauer E, Merdanovic M, Mortimer AP, Bringmann G, Reidl J. 2004. PnuC and the utilization of the nicotinamide riboside analog 3aminopyrimidine in Haemophilus influenzae. Antimicrob Agents Chemother 48: 4532-4541. doi:10.1128/AAC.48.12.4532-4541 .2004

Schattner P. 2002. Searching for RNA genes using base-composition statistics. Nucleic Acids Res 30: 2076-2082. doi:10.1093/nar/30.9 .2076

Sievers F, Wilm A, Dineen D, Gibson TJ, Karplus K, Li W, Lopez R, McWilliam H, Remmert M, Söding J, et al. 2011. Fast, scalable generation of high-quality protein multiple sequence alignments using Clustal Omega. Mol Syst Biol 7: 539. doi:10.1038/msb .2011 .75

Soukup GA, Breaker RR. 1999. Relationship between internucleotide linkage geometry and the stability of RNA. RNA 5: 1308-1325. doi:10.1017/S1355838299990891

Stav S, Atilho RM, Mirihana Arachchilage G, Nguyen G, Higgs G, Breaker RR. 2019. Genome-wide discovery of structured noncoding RNAs in bacteria. BMC Microbiol 19: 66. doi:10.1186/s12866019-1433-7

Vogl C, Grill S, Schilling O, Stülke MM, Stolz J. 2007. Characterization of riboflavin (vitamin $B_{2}$ ) transport proteins from Bacillus subtilis and Corynebacterium glutamicum. J Bacteriol 189: 7367-7375. doi:10.1128/JB.00590-07

Weinberg Z, Lünse CE, Corbino KA, Ames TD, Nelson JW, Roth A, Perkins KR, Sherlock ME, Breaker RR. 2017. Detection of 224 candidate structured RNAs by comparative analysis of specific subsets of intergenic regions. Nucleic Acids Res 18: 10811-10823. doi:10 $.1093 / \mathrm{nar} / \mathrm{gk} \times 699$

Wickiser JK, Winkler WC, Breaker RR, Crothers DM. 2005a. The speed of RNA transcription and metabolite binding kinetics operate an FMN riboswitch. Mol Cell 18: 49-60. doi:10.1016/j.molcel.2005 .02 .032

Wickiser JK, Cheah MT, Breaker RR, Crothers DM. 2005b. The kinetics of ligand binding by an adenosine-sensing riboswitch. Biochemistry 44: 13404-13414. doi:10.1021/bi051008u

Zhu N, Olivera BM, Roth JR. 1989. Genetic characterization of the pnuC gene, which encodes a component of the nicotinamide mononucleotide transport system in Salmonella typhimurium. J Bacteriol 171: 4402-4409. doi:10.1128/JB.171.8.4402-4409 .1989 

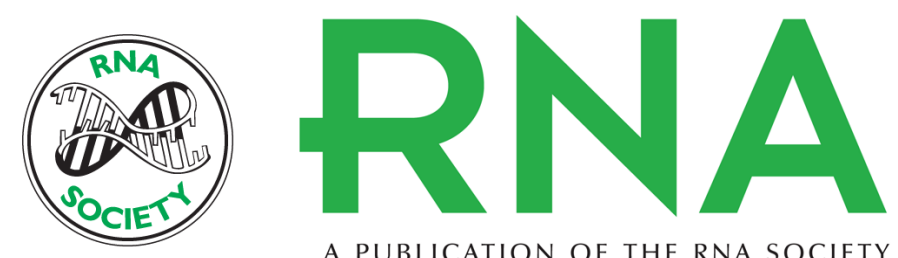

A PUBLICATION OF THE RNA SOCIETY

\title{
A second riboswitch class for the enzyme cofactor NAD+
}

\author{
Shanker S.S. Panchapakesan, Lukas Corey, Sarah N. Malkowski, et al.
}

RNA 2021 27: 99-105 originally published online October 21, 2020

Access the most recent version at doi:10.1261/rna.077891.120

\section{Supplemental http://rnajournal.cshlp.org/content/suppl/2020/10/21/rna.077891.120.DC1 Material \\ References This article cites 33 articles, 14 of which can be accessed free at: http://rnajournal.cshlp.org/content/27/1/99.full.html\#ref-list-1 \\ Open Access Freely available online through the RNA Open Access option. \\ Creative This article, published in RNA, is available under a Creative Commons License Commons (Attribution 4.0 International), as described at \\ License http://creativecommons.org/licenses/by/4.0/.}
Email Alerting Receive free email alerts when new articles cite this article - sign up in the box at the Service top right corner of the article or click here.

\section{|IIIIII Providing Precise Solutions for your research.}

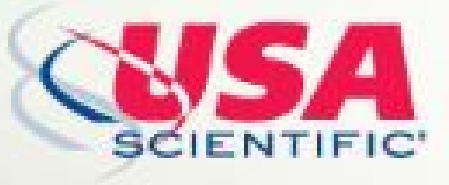

To subscribe to RNA go to:

http://rnajournal.cshlp.org/subscriptions

(C) 2021 Panchapakesan et al.; Published by Cold Spring Harbor Laboratory Press for the RNA Society 